\title{
Premetazoan genome evolution and the regulation of cell differentiation in the choanoflagellate Salpingoeca rosetta
}

\author{
Stephen R Fairclough ${ }^{1 \dagger}$, Zehua Chen ${ }^{2 \dagger}$, Eric Kramer $^{3}$, Qiandong Zeng ${ }^{2}$, Sarah Young ${ }^{2}$, Hugh M Robertson ${ }^{4}$, \\ Emina Begovic ${ }^{1}$, Daniel J Richter ${ }^{1}$, Carsten Russ ${ }^{2}$, M Jody Westbrook ${ }^{1}$, Gerard Manning ${ }^{3}$, B Franz Lang ${ }^{5}$, Brian Haas ${ }^{2}$, \\ Chad Nusbaum ${ }^{2^{*}}$ and Nicole King ${ }^{1 *}$
}

\begin{abstract}
Background: Metazoan multicellularity is rooted in mechanisms of cell adhesion, signaling, and differentiation that first evolved in the progenitors of metazoans. To reconstruct the genome composition of metazoan ancestors, we sequenced the genome and transcriptome of the choanoflagellate Salpingoeca rosetta, a close relative of metazoans that forms rosette-shaped colonies of cells.

Results: A comparison of the $55 \mathrm{Mb}$ S. rosetta genome with genomes from diverse opisthokonts suggests that the origin of metazoans was preceded by a period of dynamic gene gain and loss. The S. rosetta genome encodes homologs of cell adhesion, neuropeptide, and glycosphingolipid metabolism genes previously found only in metazoans and expands the repertoire of genes inferred to have been present in the progenitors of metazoans and choanoflagellates. Transcriptome analysis revealed that all four S. rosetta septins are upregulated in colonies relative to single cells, suggesting that these conserved cytokinesis proteins may regulate incomplete cytokinesis during colony development. Furthermore, genes shared exclusively by metazoans and choanoflagellates were disproportionately upregulated in colonies and the single cells from which they develop.

Conclusions: The S. rosetta genome sequence refines the catalog of metazoan-specific genes while also extending the evolutionary history of certain gene families that are central to metazoan biology. Transcriptome data suggest that conserved cytokinesis genes, including septins, may contribute to S. rosetta colony formation and indicate that the initiation of colony development may preferentially draw upon genes shared with metazoans, while later stages of colony maturation are likely regulated by genes unique to S. rosetta.
\end{abstract}

\section{Background}

Metazoan multicellularity and development are rooted in basic mechanisms of cell adhesion, signaling, and differentiation that were present in the unicellular and colonial progenitors of metazoans. Reconstructing the evolution of metazoans from their single celled ancestors promises to illuminate one of the major transitions in evolutionary history, while also revealing fundamental mechanisms underlying metazoan cell biology and

\footnotetext{
* Correspondence: chad@broadinstitute.org; nking@berkeley.edu

+ Contributed equally

'Department of Molecular and Cell Biology, University of California Berkeley, Berkeley, CA 94720, USA

${ }^{2}$ Broad Institute of MIT and Harvard, Cambridge, MA 02141, USA

Full list of author information is available at the end of the article
}

multicellularity. Although the first metazoans evolved over 600 million years ago, insights into their biology and origin may be gained through the comparison of metazoan genomes with those of their closest living relatives, the choanoflagellates [1-3]. Indeed, the genome of the first sequenced choanoflagellate, the single-celled species Monosiga brevicollis, provided evidence that diverse protein domains characteristic of metazoan signaling and adhesion proteins (for example, tyrosine kinase (TK), cadherin, and Hedgehog (Hh) domains) evolved before the divergence of choanoflagellates and metazoans [2].

The evolution of metazoans from their single-celled ancestors is hypothesized to have involved a transition through a colonial intermediate $[4,5]$, the Urblastea, which

\section{Biomed Central}


may have been composed of choanoflagellate-like cells [5] (Figure 1). The rosette-shaped colonies formed by the choanoflagellate Salpingoeca rosetta evoke the hypothesized Urblastea (Figure 1a, b). In addition to rosette colonies, the life history of $S$. rosetta includes diverse cell types and morphologies, including linear chains of cells ('chain colonies'), slow and fast swimmer cells, and thecate cells that attach to substrates through a secreted structure called a theca (Figure 1c) [6]. The diversity of these forms is comparable to the number of cell types observed in sponges and placozoans [7]. Therefore, sequencing the $S$. rosetta genome would provide an opportunity to investigate how genome evolution and cell differentiation in the ancestors of metazoans and multicellular choanoflagellates laid the foundations for metazoan cell biology and development. Furthermore, comparisons between the genomes of M. brevicollis and S. rosetta offer the opportunity to investigate the genetic bases of multicellularity in choanoflagellates. To these ends, we have sequenced and analyzed the $S$. rosetta genome and transcriptome during multiple key phases in the S. rosetta life history.

\section{Results and discussion}

The approximately $55 \mathrm{Mb}$ S. rosetta genome was sequenced to $33 \times$ average coverage with a combination of Sanger and 454 technology and assembled into 154 scaffolds with an N50 average length of $1.52 \mathrm{Mb}$ (Table S1 in Additional file 1). The genome assembly is largely complete, capturing approximately $96 \%$ of transcripts assembled de novo from RNA-seq data (Table S2 in Additional file 1). Predicted telomeres were found at both ends of 21 scaffolds and 24 additional scaffolds contain a single telomeric end, suggesting that $S$. rosetta has a minimum of 33 chromosomes (Table S3 in Additional file 1). A starting set of $a b$ initio gene predictions generated by the Broad Institute annotation pipeline trained with ESTs (generated by Sanger chemistry) was refined using $21 \mathrm{~Gb}$ of transcriptome sequence (generated by Illumina chemistry) collected from diverse life history stages (Additional file 1, Figure S1). This gene catalog contains 11,629 genes, of which $98 \%$ are supported by transcriptome sequence data (Table S1 in Additional file 1 ). Aligning the protein sequences from this gene set to the $M$. brevicollis protein set revealed 4,994 orthologous pairs, yet the two species display relatively little gene synteny (Figure S14 in Additional file 1).

To reconstruct the gene contents of the progenitors of metazoans and choanoflagellates, we compared the genomes of S. rosetta and M. brevicollis [2] with the sequenced genomes of 32 representative metazoans and metazoan outgroups (Table S4 in Additional file 1). Evolutionary relationships among genes from different genomes were predicted using OrthoMCL2 [8] to identify 'ortholog clusters' (Additional files 2 and 4). The 11,629 genes of $S$. rosetta fall into 9,411 ortholog clusters (that is, some ortholog clusters contain multiple $S$. rosetta genes). The evolutionary history of each ortholog cluster was inferred by mapping its distribution onto a reference phylogeny (Figure 1a), allowing us to gain insight into the composition of ancestral genomes and patterns of gene gain and loss in the lineages leading to metazoans, choanoflagellates, and fungi (Figure 2; Additional file 5). Gene families and protein domains of particular interest were also curated manually (see, for example, Figures S7 to S9, S13 and Tables S6 and S8 in Additional file 1).

The genomes of the ancestors ('Ur-'; Figure 1a) of metazoans and opisthokonts (metazoans + choanoflagellates + fungi) were each predicted to have contained members of about 10,000 ortholog clusters. While nearly $20 \%(1,843)$ of the ortholog clusters from the Uropisthokont were lost along the lineage leading to the Urmetazoan, this lineage also experienced an equivalent amount of gene gain (Figure 2). In contrast, fungi and choanoflagellates apparently lost representation from $33 \%(3,372)$ and $47 \%(4,747)$ of the ancestral Uropisthokont ortholog clusters, respectively, but experienced only half as much gene gain. The $S$. rosetta genome also reveals that $M$. brevicollis has lost an additional 1,343 genes. Therefore, the $S$. rosetta genome sequence substantially clarifies the gene content of the Urchoanimal. Future sequencing of additional choanoflagellate genomes will further refine inferences about the gene content of the Urchoanimal, presumably by reducing the number of genes thought to be metazoan. Nonetheless, patterns of gene gain and loss based on currently sequenced genomes speak to the richness of the gene complement in the Uropisthokont [3] and emphasize the role that gene birth may have played in the evolution of biological novelties such as metazoan multicellularity.

Therefore, we next characterized the 5,706 ortholog clusters that appear to have evolved along the stem lineage leading to metazoans (Additional file 3). These metazoan-specific ortholog clusters include homologs of genes that regulate cell adhesion, including $\delta$-catenin and $\beta$-laminin, as well as genes involved in the transforming growth factor (TGF) $-\beta$ and Wnt developmental signaling pathways. Many of the core components of the TGF- $\beta$ and Wnt signaling pathways (for example, TGF$\beta$, TGF- $\beta$ receptor, Smad, Wnt, Wntless, $\beta$-catenin, and TCF) were identified in every metazoan genome included in our analysis, underscoring their early evolution and fundamental importance to metazoan biology.

Genes shared between choanoflagellates and metazoans were present in the progenitors of metazoans and may have contributed to the genomic foundations of the origin of metazoans. We find that the evolution of the 


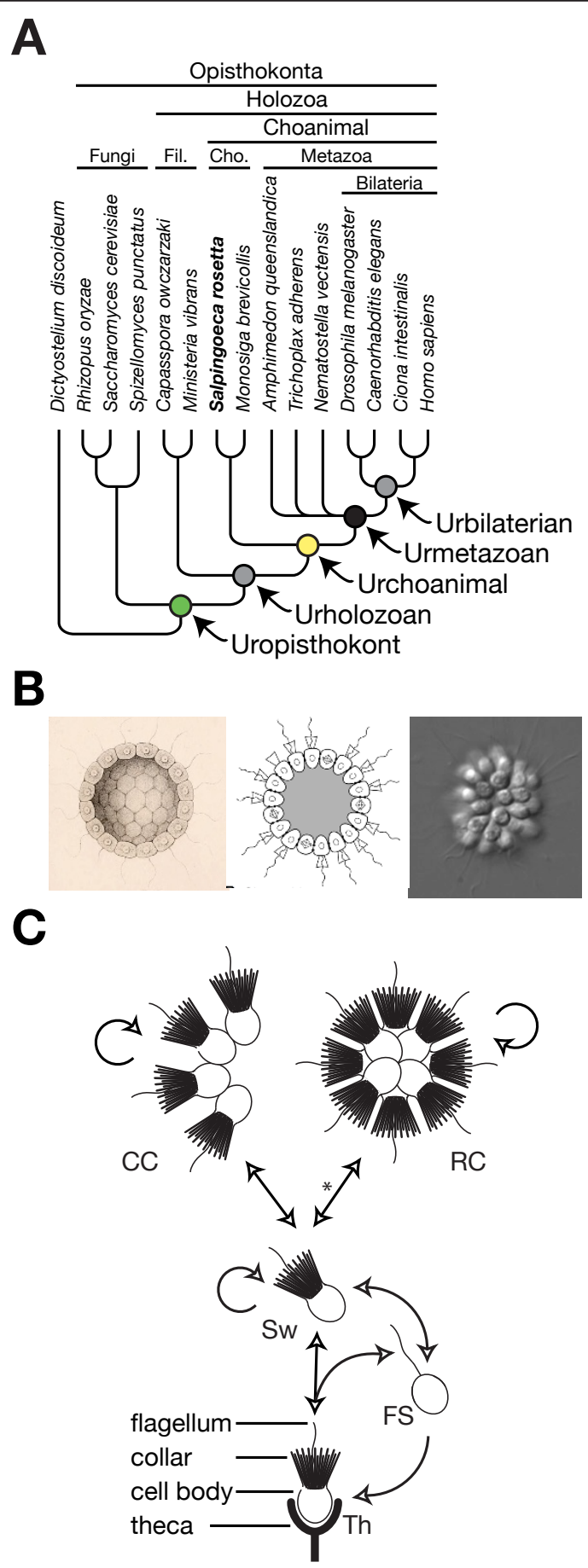

Figure 1 Salpingoeca rosetta as a model for studying the ancestry of metazoan multicellularity. (a) Choanoflagellates are the closest living relatives of Metazoa $[1,3,68]$. Taxonomic groupings are indicated above the phylogeny and the last common ancestors of each group are indicated as colored circles at nodes. The topology of the reference phylogeny was based on a consensus of results from [68-70]. Branches were collapsed at the base of Metazoa to reflect current uncertainty about the identity and branch order of the most basal metazoan phyla [69,71-73]. The black, yellow, and green colored nodes are used in both Figures 1 and 2 to represent the Urmetazoan, Urchoanimal, and Uropisthokont, respectively. (b) The evolution of metazoans from their single-celled ancestors is hypothesized to have involved a transition through a simple colonial form, such as Haeckel's Blastea (left, from Figure 117 of [4]) or Nielsen's Choanoblastea (center, from [5]), that resembles the rosette colonies formed by S. rosetta (right). (c) S. rosetta can transition through at least five morphologically and behaviorally differentiated cell types [6]. Solitary 'thecate' cells attached to a substrate (Th) can produce solitary swimming (Sw) cells or solitary fast swimming (FS) cells, either through cell division or theca abandonment. Solitary swimming cells can divide completely to produce solitary daughter cells or remain attached after undergoing incomplete cytokinesis to produce either chain colonies (CC), or rosette colonies (RC) in the presence of the bacterium Algoriphagus machipongonensis (asterisk) [6,18,64]. Fil., Filasterea; Cho., Choanoflagellates. 


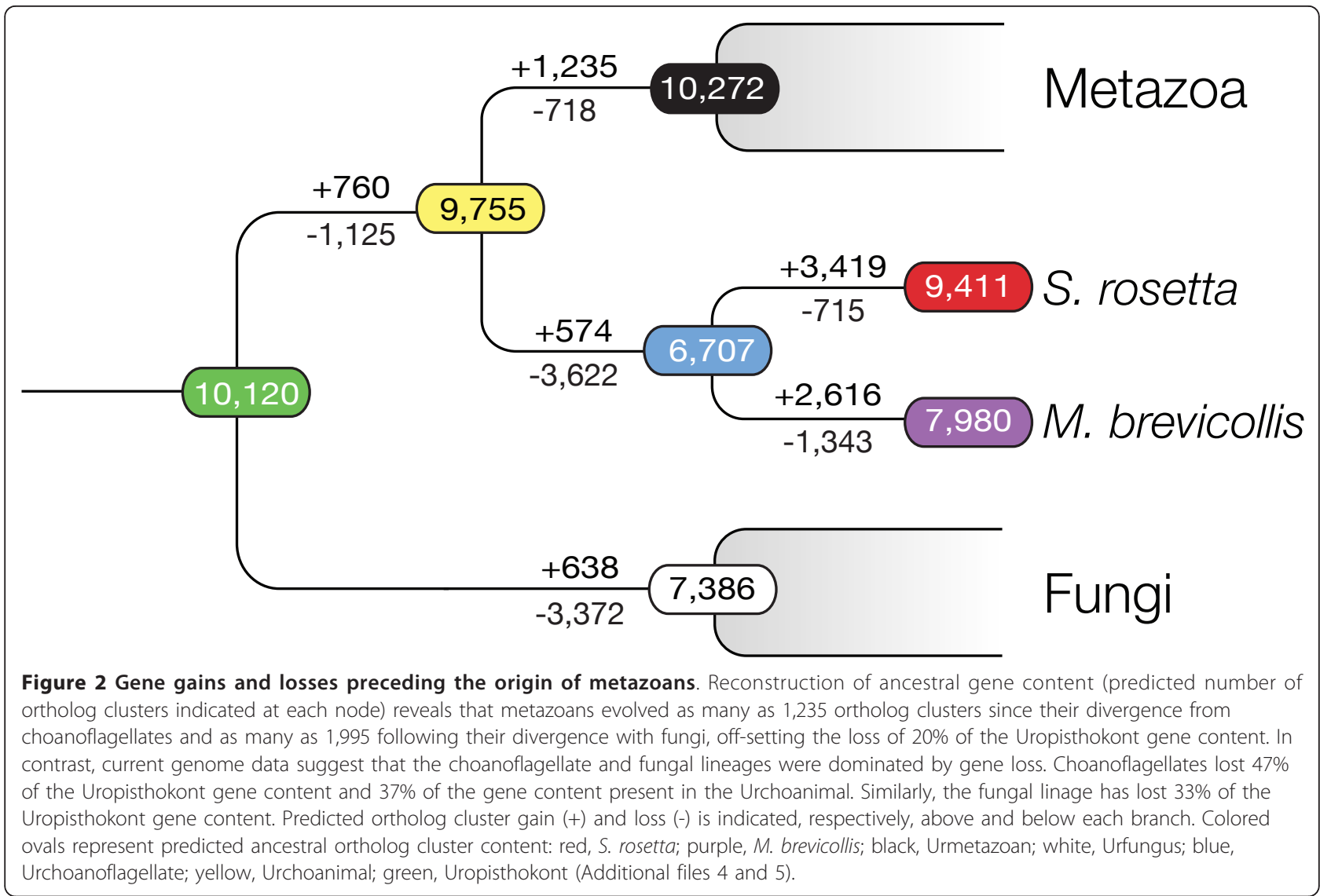

monophyletic 'Choanimal' clade (which contains choanoflagellates and metazoans, and is not to be confused with the paraphyletic 'Choanozoa' (see Endnote a)), was marked by a disproportionate gain of genes with Gene Ontology terms [9] for metazoan cell adhesion and cell-junction organization (Table S5 in Additional file 1), including cadherins, PATJ (a component of adherens junctions) and KANK/vab-19 (an ankyrin repeat protein required for proper embryonic epidermal elongation and muscle attachment to the epidermis in Caenorhabditis elegans [10]). Ortholog clusters involved in metazoan neuropeptide signaling and glycosphingolipid metabolism also increased in abundance (Table S5 in Additional file 1). In addition, the $S$. rosetta genome, like that of $M$. brevicollis, contains a diverse and abundant repertoire of TKs (see Endnote b) $[2,11,12]$ (Additional file 6). Ninety percent of the $S$. rosetta cytoplasmic TKs are conserved in the $M$. brevicollis genome, and $S$. rosetta has homologs of two adhesion-associated cytoplasmic TKs, FAK and Fer, that were apparently lost in M. brevicollis (Table S6 in Additional file 1). In contrast, only $21 \%$ of receptor TKs (RTKs) from $S$. rosetta and $M$. brevicollis form orthologous pairs. The added sequence diversity provided by the $S$. rosetta genome also revealed that choanoflagellates may have divergent homologs of metazoan Eph RTKs that were not originally detected in the $M$. brevicollis genome. Eph RTKs are key regulators of cell migration during development, regulating cellular organization through differential cell repulsion and adhesion [13]. Their discovery in S. rosetta lays the foundation for investigating core and ancestral functions of these important receptors.

The $S$. rosetta genome now provides a platform for investigating the regulation of cell differentiation in choanoflagellates and the potential evolutionary connections between the cell biology of choanoflagellates and metazoans. Therefore, we analyzed the transcriptional profiles of samples enriched in each of four different $S$. rosetta cell types: thecate cells, swimming cells (a mix of slow and fast swimmers), chain colonies, and rosette colonies (Figure 1c; Figure S1 in Additional file 1; Additional file 7). Using three independent analytical approaches we identified $480 \mathrm{~S}$. rosetta genes that were consistently upregulated in colonies (chains and rosettes) compared to solitary cells (swimming and thecate) and 1,410 genes that were consistently upregulated in thecate cells relative to swimming solitary cells and colonies (Figures S2, S3 and S4 in Additional file 1; Additional files 8 to 13). For example, in colonies and thecate cells distinct subsets of TKs, cadherins (notable for their roles in metazoan cell signaling and adhesion) 
and Hh-domain containing proteins (see Endnote c) were significantly upregulated (Figures S5, S6, and S12 in Additional file 1), although their functions in these contexts are unknown.

Perhaps most illuminating was the observation that all four members of the $S$. rosetta septin gene family were significantly upregulated in colonies (Figure 3a). Septins, conserved GTPases that regulate cytokinesis in fungi and metazoans, were first identified in yeast through a screen for cytokinesis defects; septin mutants frequently failed to undergo proper cytokinesis and therefore exhibited multicellular phenotypes [14]. Metazoan septins also stabilize intercellular bridges such as midbodies and ring canals $[15,16]$. During metazoan cytokinesis and in intercellular bridges, a set of specific septin monomers polymerize to form cytoskeletal filaments [17]. The four S. rosetta septins have conserved amino acid residues on predicted interacting surfaces, suggesting they may also form filaments (Figure 3b; Figures S7 and S8 in Additional file 1). Interestingly, S. rosetta homologs of other midbody-associated proteins and septin regulators, including Aurora kinase, the scaffolding protein Anillin, and Polo kinase, are also significantly upregulated in colonies (Figure 3a). The coordinated upregulation of septins and septin regulators is notable because colony development in S. rosetta occurs by incomplete cytokinesis, such that neighboring cells remain physically linked by intercellular bridges (Figure 3c) $[6,18]$.

The genes that regulated cell differentiation in the progenitors of metazoans may have provided the foundations for the spatiotemporal regulation of cell differentiation that underpins metazoan development. Therefore, understanding the evolutionary history of genes differentially expressed in different $S$. rosetta cell types may suggest which cell types are most conducive to the study of metazoan origins. Of the 11,628 genes in the S. rosetta genome, at least $57 \%$ were present in the Uropisthokont, $5 \%$ arose on the Urchoanimal stem, $6 \%$ are choanoflagellate-specific, and $31 \%$ are apparently unique to S. rosetta (Figure 4a; Table S7 in Additional file 1). The evolutionary histories of genes upregulated in specific cell types deviated significantly from this distribution. For example, thecate cells disproportionately upregulated genes that evolved within choanoflagellates, after their divergence from the metazoan stem lineage (Figure 4b; Table S7 in Additional file 1). Therefore, the unusual morphology and transcriptional profile of thecate cells suggest that important aspects of their biology may be unique to choanoflagellates. Colony development, in contrast, has potential relevance for understanding the regulation of early metazoan multicellularity. Colonies develop from a subset of solitary swimming cells [6], so the most likely regulators of colony development are the 352 genes that are specifically upregulated in both solitary swimming cells and in colonies (Figure 4c; Table S7 in Additional file 1). Interestingly, this set is highly enriched in genes that are exclusively shared with metazoans and that presumably evolved along the Urchoanimal stem lineage. Genes involved in the maintenance of mature colonies, as opposed to those involved in regulating early colony development, would be expected to be specifically upregulated in colonies (Figure 4d; Table S7 in Additional file 1), but not in the single cells from which they develop (Figure 4e; Table S7 in Additional file $1)$. This set was enriched in genes unique to $S$. rosetta. Taken together, these data led us to hypothesize that the initiation of $S$. rosetta colony development draws upon genes shared with metazoans, while later stages of colony maturation are regulated by genes that are unique to $S$. rosetta.

\section{Conclusions}

Although the progenitors of metazoans expired over 600 million years ago $[19,20]$, genome comparisons between metazoans and their closest relatives, the choanoflagellates, can offer detailed insights into the evolutionary foundations of metazoan genomes and gene families [20]. The $S$. rosetta genome refines the catalog of metazoanspecific genes and highlights the potential relevance of key gene families to the evolution of defining features of metazoan biology. Genes with a variety of evolutionary histories - including highly conserved genes with functions that are integral to eukaryotic cell biology, genes that evolved before the choanimals and that were subsequently co-opted to new metazoan-specific functions, and new genes whose evolution may have served as key innovations - shaped the evolution of metazoans from their protistan ancestors [21,22]. With this more complete gene catalog, it is now possible to reconstruct the ancestry of metazoan gene families in unprecedented detail (for example, Figures S6 and S9 in Additional file 1 , and $[23,24])$. The $M$. brevicollis genome sequence previously revealed that diverse protein domains in metazoan signaling and adhesion genes, including cadherin, $\mathrm{Hh}$, and TK, evolved before the origin of metazoan multicellularity [2]. The $S$. rosetta genome now reveals that the Urchoanimal genome contained representatives of at least eight metazoan TK gene families (Table S6 in Additional file 1), including the developmentally important Eph RTKs, and raises questions about their ancestral functions in the Urchoanimal $[25,26]$.

In addition to expanding gene family representation, the S. rosetta genome also sheds light on the pathways in which these genes are traditionally thought to operate. For example, while some components of the TK and Hh developmental signaling pathways are conserved in choanoflagellates (for example, Src, Eph RTK, and Patched), others are not. Therefore, the evolution of these pathways 


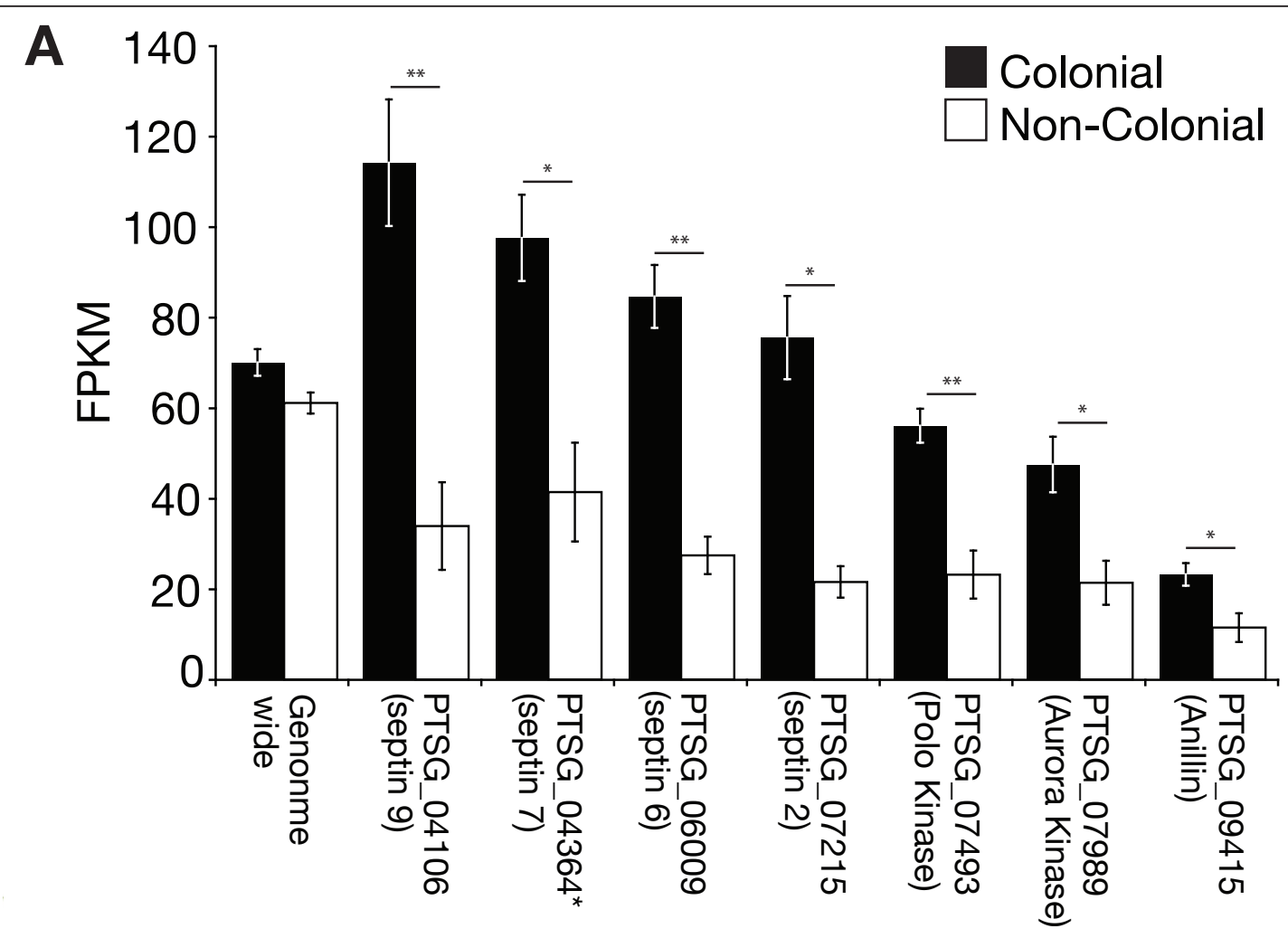

B
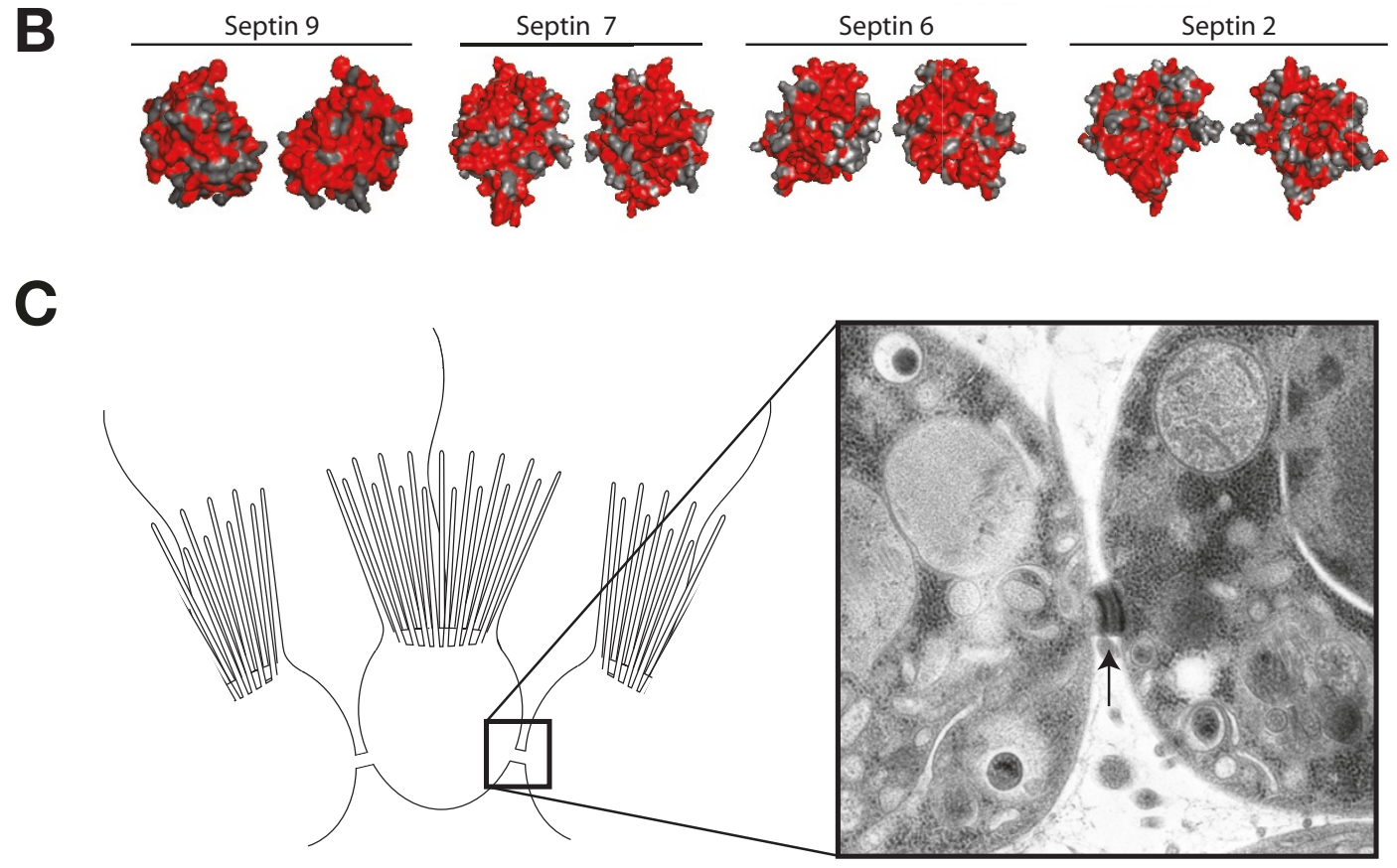

Figure 3 Septins are upregulated in colonial cells. (a) Unlike the average for all genes in the genome, septins, the septin-associated Polo and Aurora kinases, and Anillin are each significantly upregulated in colonial cells. FPKM, fragments per kilobase per million reads. Error bars are

standard error: ${ }^{*} P<0.05,{ }^{* *} P<0.01$. (b) Conserved and similar residues shared between S. rosetta septins and human septins (red) on monomer surfaces predicted to interact in human septin filaments [74] suggest that S. rosetta septins also form filaments. (c) Septins regulate cytokinesis in metazoans and fungi $[14,16]$, providing a potential connection to the narrow intercellular bridges (arrowhead), likely formed through incomplete cytokinesis, that connect neighboring cells in S. rosetta colonies. 

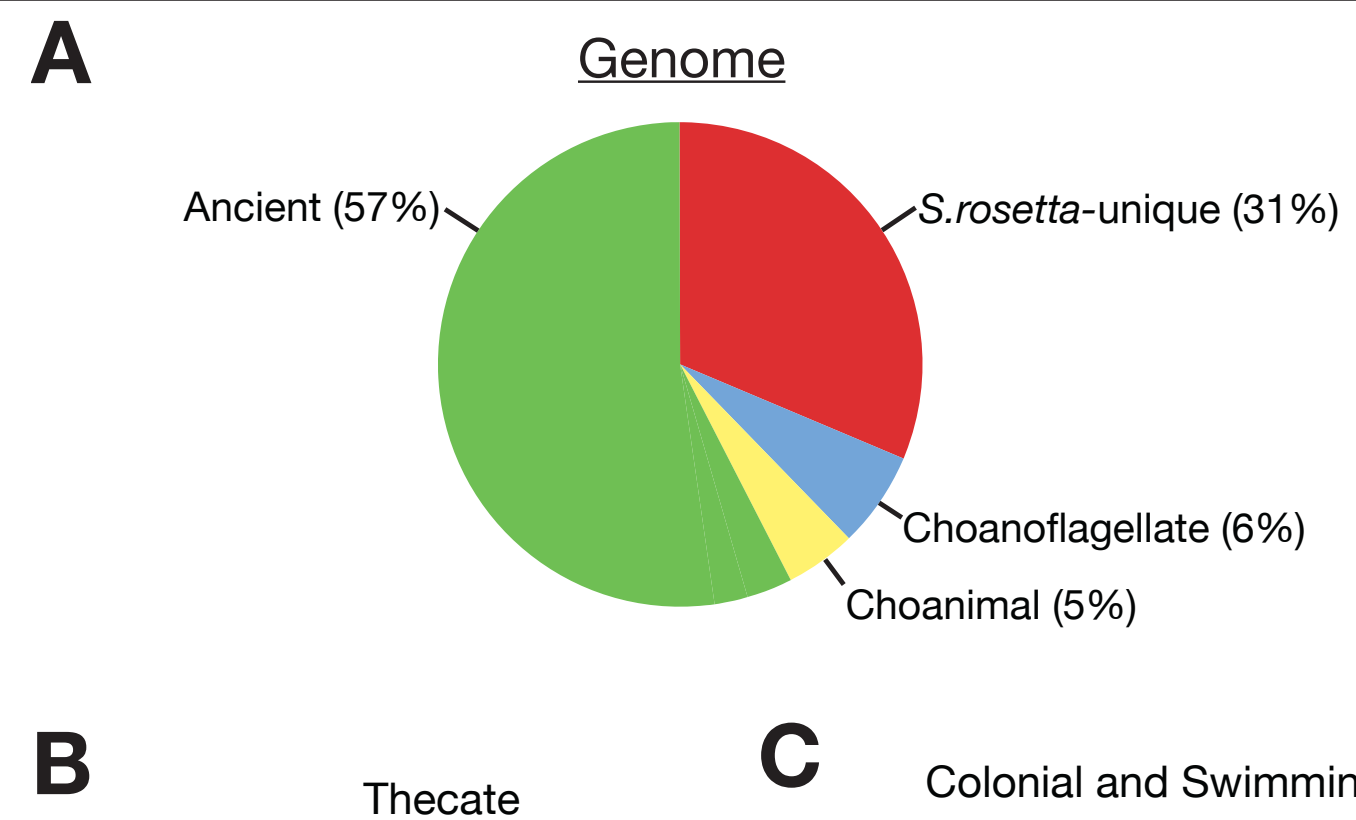

\section{Colonial and Swimming}
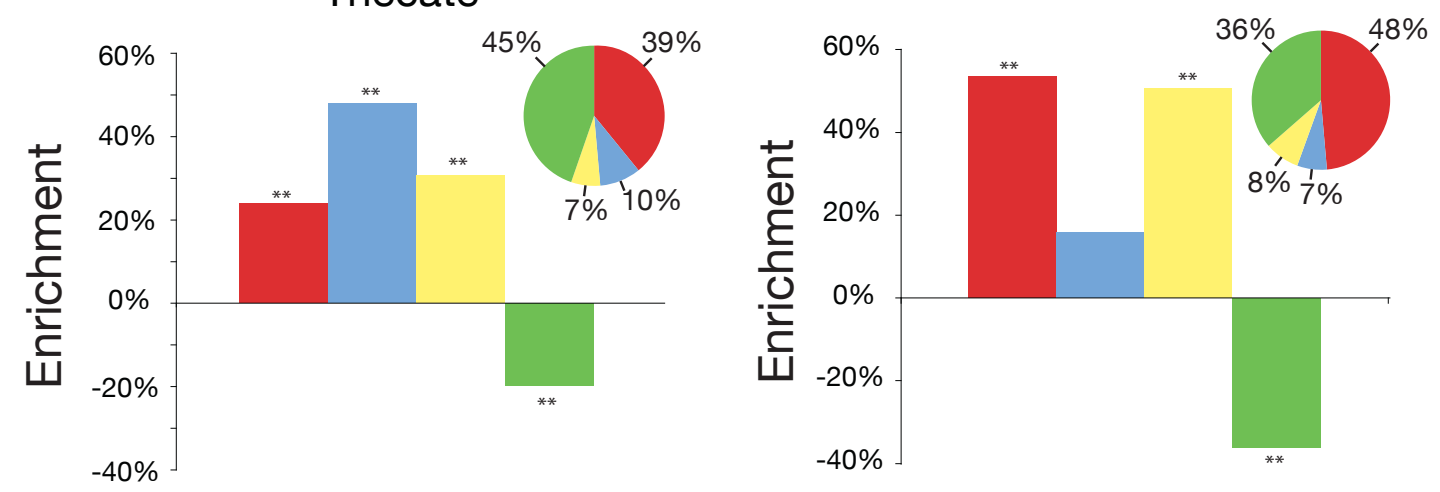

D
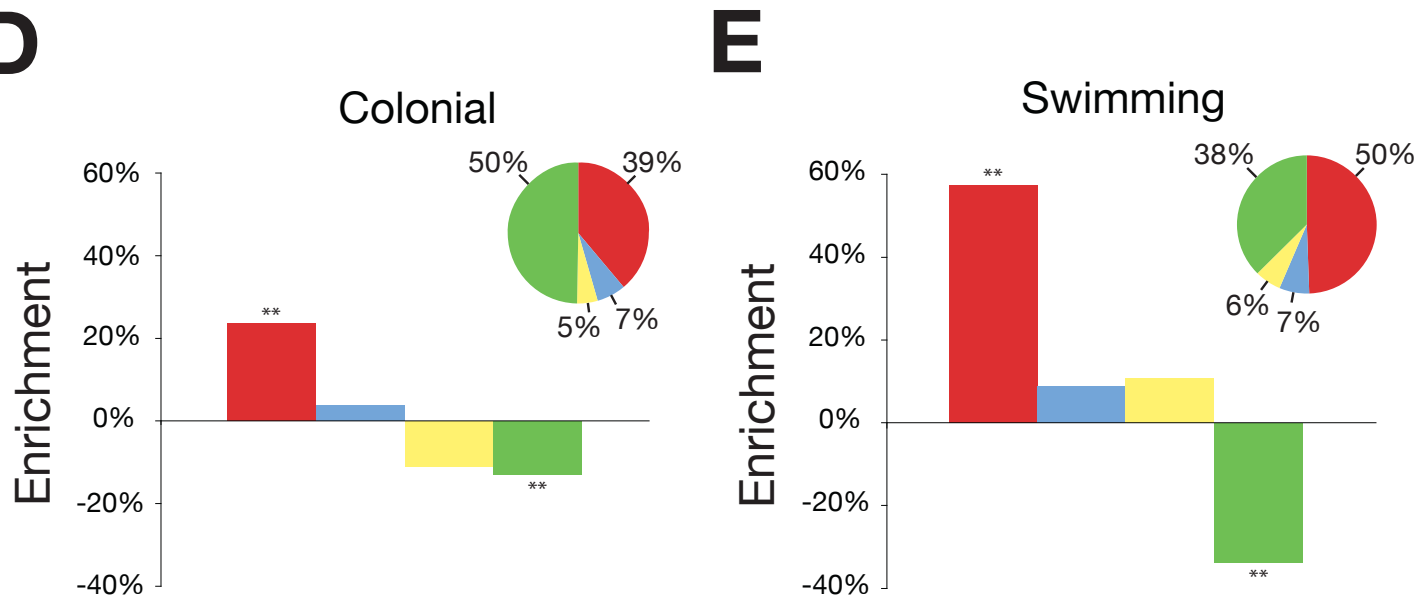

Figure 4 Different S. rosetta cell types disproportionately upregulate genes with different evolutionary histories. (a) The majority (57\%) of S. rosetta genes are ancient and evolved prior to the divergence of choanoflagellates, metazoans and fungi. An additional $5 \%$ of S. rosetta genes emerged along the stem lineage leading to S. rosetta and metazoans and $6 \%$ evolved along the choanoflagellate stem lineage. Thirty-one percent of genes in the $\mathrm{S}$. rosetta genome are apparently unique to $\mathrm{S}$. rosetta. (b-e) The evolutionary history of $\mathrm{S}$. rosetta genes upregulated in different cell types (pie charts) and the percent enrichment (y-axis) relative to the S. rosetta genome (bar graphs). Color code: red, S. rosettaspecific genes; blue, genes restricted to choanoflagellates; yellow, genes uniquely shared by choanoflagellates and metazoans; green, genes restricted to opisthokonts. (b) Thecate cells. (c) Colonies and swimming cells. (d) Colonies (rosettes and chains). (e) Swimming cells. ${ }^{* *} P<0.01$. 
along the metazoan stem lineage likely involved an as-yet undefined combination of protein domain shuffling, gene cooption, and evolution of new protein-protein interactions $[2,27]$ that promises to be further elucidated by the continued study of diverse early-branching metazoans, choanoflagellates and other metazoan outgroups $[24,28]$. In contrast, components of the Wnt pathway have not been identified in any sequenced non-metazoan genome, including those of S. rosetta and M. brevicollis, suggesting that the pathway did not evolve until after the divergence of metazoans and choanoflagellates [29-31]. A sponge classical cadherin has proven capable of binding in vitro [23] to its cognate $\beta$-catenin, a Wnt pathway effector, suggesting that at least a portion of the critical interactions in the Wnt pathway evolved before the Cambrian radiation. Given the ubiquity of Wnt pathway components in metazoans and their essential roles in regulating embryonic patterning in diverse animals, it is therefore possible that the evolution of the Wnt pathway was critical to the early evolution of metazoans.

Finally, the S. rosetta genome offers the opportunity to investigate whether choanoflagellate colony formation and metazoan development are regulated by conserved mechanisms. The upregulation of conserved genes and gene families in colonies, such as septins, cadherins, and Hh-related proteins, is intriguing and warrants further investigation to fully understand their current and ancestral functions. Taken together, the S. rosetta genome and transcriptome suggest that the genome of the last common ancestor of choanoflagellates and metazoans contained genes and domains that orchestrate development in modern animals but underwent important changes in gene content and regulation en route to the evolution of the first metazoan. Further refinement of ancestral genomes through comparative genomics with additional choanoflagellate genomes and functional efforts in choanoflagellates and sponges promises to reveal the minimal set of genes required for metazoan development and multicellularity.

\section{Materials and methods}

\section{Salpingoeca rosetta culture conditions}

$S$. rosetta, a colonial choanoflagellate originally isolated from Hog Island, Virginia, was cultured with co-isolated bacteria at $25^{\circ} \mathrm{C}$ in natural seawater infused with cereal grass media [32]. The strain sequenced in this study is deposited at the ATCC under strain number ATCC PRA-366.

\section{Isolation of S. rosetta genomic DNA}

Genomic DNA was harvested from a monoxenic culture of $S$. rosetta in which the sole source of bacteria was Algoriphagus machipongonensis [6]. S. rosetta DNA was separated from the A. machipongonensis DNA on a
$\mathrm{CsCl}$ gradient as described for the genome sequencing of $M$. brevicollis [2].

\section{Genome sequencing}

Purified S. rosetta genomic DNA was sequenced with 454 and Sanger Whole Genome Shotgun methodology as described below.

\section{4 sequencing}

454 fragment and approximately $3 \mathrm{~kb}$ jumping libraries were generated as previously described [33]. In short, $S$. rosetta genomic DNA was sheared into small fragments, approximately 600 bp for fragment and approximately 3 $\mathrm{kb}$ for jumping libraries. For fragment library construction DNA was ligated on both ends with 454 sequencing adapters. For $3 \mathrm{~kb}$ jumping library construction, DNA was ligated with biotinylated adapters on both ends to facilitate circularization. Adapted DNA was circularized, sheared and resulting fragments were ligated on both ends with 454 sequencing adapters. Library fragments containing biotin were retrieved using streptavidin beads. Both library types were subjected to emulsion PCR and sequenced with approximately 400 base titanium chemistry reads using a 454 GS FLX instrument following the manufacturer's recommendations (454 Life Sciences/Roche, Branford, Connecticut, USA).

\section{Sanger sequencing}

Genomic DNA was sheared and cloned into plasmid (4 kb and $10 \mathrm{~kb}$ insert) and Fosmid (40 kb) vectors using standard methods. Resulting whole genome shotgun libraries were paired-end Sanger sequenced using standard methods.

\section{Genome assembly}

454 data were first assembled using 454's Newbler assembler [34]. 454 assembly was then combined with Sanger data using the HybridAssemble [35] module of the ARACHNE assembler [36]. The assembly was then manually modified to close additional gaps and break misassembled joins using ARACHNE tools.

\section{Telomere identification}

Six supercontigs containing telomeric ends (Table S3 in Additional file 1, fourth column) were identified by searching the genome assembly for TTAGGG repeats. Examination of the subtelomeric regions of these six supercontigs did not reveal genes that are shared at the other telomeres below, so they appear to be aberrant or newly formed telomeres without the subtelomeric repeated regions of most telomeres.

Additional telomeric supercontigs were identified by searching the raw reads from the approximately $40 \mathrm{~kb}$ insert fosmids with 1,000 bases of TTAGGG repeats. The mate pairs of the first 250 such hits, all of which were in plus/minus arrangement, indicating that they 
were from telomeres, were then searched against the supercontigs to identify telomeric supercontigs. This search revealed 41 such supercontigs (fifth column of Table S3 in Additional file 1), including four of the six with assembled TTAGGG repeats. Clearly this is an underestimate of the number of telomeres, both because only four of the six assembled ones were identified, and because this search yields a Poisson distribution of such hits (fifth column of Table S3 in Additional file 1), ten of which were only hit once. From the average positions of the mate-pair hits within each supercontig it was possible to estimate the length of DNA missing between the assembly and the TTAGGG repeats of the telomere, and this is shown in the sixth column of Table S3 in Additional file 1.

Examination of these 37 telomeric supercontigs without assembled TTAGGG repeats revealed that all but a few of them have regions repeated on most of the others (seventh column in Table S3 in Additional file 1). The few exceptions are instances where the gap between the end of the supercontig and the TTAGGG repeats is near the $40 \mathrm{~kb}$ insert size of the fosmids, so presumably the shared subtelomeric regions are within this missing part. This approach allowed us to discover 22 additional telomeric supercontigs.

\section{Genome annotation}

Protein-coding genes were initially annotated using a combination of $a b$ initio predictions (GeneMark.hmmES, AUGUSTUS, GlimmerHMM), protein sequence homology-based evidence (blast, GeneWise), and transcript structures built from ESTs using the PASA package [37]. The package EVM (EVidenceModeler) [38] was used to build gene models from all available input evidence. The obtained gene models were further improved by incorporating RNAseq data from eight different conditions using PASA and inchworm pipelines to get a final gene set $[39,40]$. Gene models were also annotated with gene ontology terms using Blast2Go (Additional file 14) and interPro2GO (Additional file 15 ), and gene ontology enrichment was measured with Ontologizer 2.0 using default settings correcting for multiple testing.

\section{Synteny analysis}

Protein sequences from S. rosetta and M. brevicollis were aligned using BLAST [41].

Best reciprocal BLAST pairs with a score cutoff of 75 were considered orthologs.

Predicted protein orthologs were mapped back to their genomic loci using BLAT [42] and plotted against the scaffolds with R to investigate synteny between the $S$. rosetta and $M$. brevicollis genomes.

\section{Tyrosine kinase annotation}

Manual annotations for the $S$. rosetta kinases were made through BLAST [41], multiple sequence alignments, hidden Markov models, presence or absence of accessory domains and phylogenetic trees. S. rosetta kinases were compared to nine previously annotated kinomes: Homo sapiens [43], Mus musculus [44], Strongylocentrotus purpuratus [45], Drosophila melanogaster [46], C. elegans [47], Amphimedon queenslandica [30], Monosiga brevicollis [48], Saccharomyces cerevisiae [49], and Selaginella moellendorffii [50].

\section{Septin characterization}

The final gene predictions for the $S$. rosetta genome included five septin domain encoding genes (PTSG_04106, PTSG_06009, PTSG_07215, PTSG_04363 and PTSG_04364) as predicted by Pfam [51]. A gap in the assembly suggested that PTSG_04363 and PTSG_04364 might be one gene. PCR amplification from a $S$. rosetta cDNA library using specifically designed primers (5'TCAACGAAACGATTTCAAGC and 5'GTGGTCCG AGTTGTCGACTT) confirmed this and the two gene models were merged into a new gene model (PTSG_ 04364:) (Figure S7 in Additional file 1). Conserved septinspecific residues, including the amino-terminal polybasic region, were identified manually while coiled-coil domains were predicted using the COILS program using the default settings [52]. Sequences with average probabilities below 0.8 were not considered to have coiled-coil domains (Figure S8 in Additional file 1).

\section{Septin structure prediction}

The structure of each $S$. rosetta septin was predicted using LOOPP (version 4.0) available through the University of Texas $[53,54]$. Individual $S$. rosetta septin structures were loaded into MacPymol [55] and similar residues determined using NCBI BLAST [41] alignment and colored red. Each structure was then aligned to the crystal structure of the human septin filament (accession 2QAG in the Protein Data Bank).

\section{Phylogenetic analyses}

The four $S$. rosetta septin sequences were added to a septin alignment from Momany et al. [56] in order to establish putative gene homology assignments (Figure S8 in Additional file 1). The sequences were aligned using the Clustal Omega multiple sequence alignment program [57] and variable sequence regions were systematically removed using Gblocks [58] with the most lenient parameters: Minimum Number Of Sequences For A Conserved Position, $81(\mathrm{~b} 1=81)$; Minimum Number Of Sequences For A Flanking Position, 81 (b2 = 81); Maximum Number Of Contiguous Nonconserved Positions, 
8 (b3 = 8); Minimum Length Of A Block, $5(\mathrm{~b} 4=5)$; Allowed Gap Positions, With Half (b5 = h); Use Similarity Matrices, Yes $(\mathrm{b} 6=\mathrm{y})$; New number of positions, 210 (15\% of the original 1,360 positions). A maximum likelihood analysis was performed on the resulting alignment of 183 amino acid characters using PHYML v.3.0 [59]. The WAG substitution model [60] was implemented with a mixed model of rate heterogeneity and four rate categories where the fraction of invariable sites and the gamma distribution parameter alpha were estimated from the data set. Bootstrap support (100 replicates) was estimated for the single resulting tree topology (Figure S9 in Additional file 1).

\section{Reconstructing gene gain and loss in opisthokonts}

To characterize how gene content changed during the evolution of the opisthokonts, ortholog clusters were mapped to a reference phylogeny [61,62] using a Dollo parsimony model of evolution [63] and the minimal gene content at each node and the change along the subsequently diverging lineages was estimated.

\section{Cell type enrichment}

Solitary swimming $(\mathrm{Sw})$ cells were isolated from the supernatant fraction of cultures grown in the presence of mixed bacteria, but not A. machipongonensis [18].

Thecate (Th) cells were collected from cultures by removing the supernatant, washing three times with 10 $\mathrm{ml}$ of culture media and removing the attached cells from the plate surface with a plastic cell lifter.

Cultures consisting primarily of chain colonies (CC) were generated by diluting $2 \mathrm{ml}$ of cells from the supernatant of solitary swimming ( $\mathrm{Sw}$ ) cells into $15 \mathrm{ml}$ fresh medium every day for 1 to 2 weeks.

Cultures consisting primarily of rosette colonies (RC) were produced using two different strategies. In the first approach, a culture of solitary swimming (Sw) cells was inoculated with live A. machipongonensis bacteria [18], which induces the development of rosette colonies (RC) that became the dominant form in the culture within 2 days $[6,18,64]$. Rosette colonies (RC) were also isolated from cultures grown exclusively with live A. machipongonensis [6].

\section{RNAseq}

Total RNA was isolated from $S$. rosetta cultures using the RNAeasy (Qiagen, Venlo, The Netherlands) kit and four consecutive rounds of oligo-dT hybridization, washing, and elution with Oligotex kit (Qiagen) were used to purify mRNA. Purified mRNA was treated with Ambion Turbo DNA-free (Life Technologies, Carlsbad, California, USA) per the manufacturer's recommendation. The integrity of the mRNA was assessed using an Agilent 2100 Bioanalyzer (Agilent Technologies, Santa
Clara, California, USA) and quantified using RNA Quant-it assay for the Invitrogen Qubit Fluorometer (Life Technologies, Carlsbad, California, USA).

Strand specific dUTP Illumina RNA-seq libraries were generated from 200 ng mRNA as previously described [65] with the following modifications. mRNA was fragmented in $1 \times$ fragmentation buffer (Affymetrix, Santa Clara, California, USA) at $80^{\circ} \mathrm{C}$ for 4 minutes, purified and concentrated to $6 \mu \mathrm{l}$ following ethanol precipitation. Illumina sequencing adapters containing 8-base barcodes were ligated to each sample, enabling pooling of libraries. Adaptor ligation was done with $1.2 \mu \mathrm{l}$ of barcoded Illumina adaptor mix and 4,000 cohesive end units of T4 DNA Ligase (New England Biolabs, Ipswich, Massachusetts, USA) overnight at $16^{\circ} \mathrm{C}$ in a final volume of $20 \mu \mathrm{l}$. Final library insert size ranged from 225 to 425 bp. Libraries were sequenced with 68 base paired-end reads on an Illumina GAII instrument (Illumina, San Diego, California, USA) following the manufacturer's recommendations.

\section{Identification of differentially expressed genes Pairwise comparison}

To identify genes differentially expressed in a particular cell type and control for environmental variation, we compared gene expression in different fractions of the same culture. All genes identified by this method have a statistically significant difference in at least $30 \%$ of the comparisons, with the remaining comparisons showing the same trend.

Colonial versus thecate Read count was compared between samples (RCA1 versus ThA2, RCA2 versus ThA2, RCAM versus ThAM, RCAM versus ThM, CCM versus ThA2, CCM versus ThAM, CCM versus ThM) using edgeR installed under Bioconductor v2.8 and a gene was considered differentially expressed between colonial cells and attached cells if it was significantly differentially expressed $(P$-value $<0.05)$ in at least three comparisons and had a fold change greater than 1.5 in the remaining comparisons.

Colonial versus swimming Read count was compared between samples (RCA1 versus SwM, RCA2 versus SwM, RCAM versus SwM, CCM versus SwM) using edgeR installed under $\mathrm{R}$ Bioconductor v2.8 [66] and a gene was considered differentially expressed between colonial cells and swimming cells if it was significantly differentially expressed $(P$-value $<0.05)$ in at least two comparisons and had a fold change greater than 1.5 in the remaining comparisons.

Attached versus swimming Read count was compared between samples (ThA2 versus SwM, ThAM versus SwM, ThM versus SwM) using edgeR installed under $R$ Bioconductor v2.8 [66] and a gene was considered differentially expressed between attached cells and swimming 
cells if it was significantly differentially expressed ( $P$-value $<0.05$ ) in at least one comparison and had a fold change greater than 1.5 in the remaining comparisons.

\section{Group comparison}

RNAseq read count was compared between groups of samples using edgeR installed under R Bioconductor V2.8 [66] and genes are considered differentially expressed with $P$-value $<0.05$. The comparisons include: Colony versus thecate (RCA1, RCA2, RCAM, CCM versus ThA2, ThAM, ThM); Colony VS Swim (RCA1, RCA2, RCAM, CCM versus SwM); Thecate versus Swim (ThA2, ThM, ThAM versus SwM).

\section{Hierarchical clustering}

FPKM values (fragments per kilobase per million reads) for each gene were $\log 2$ transformed, quantile normalized, and filtered requiring $\operatorname{Max}(\log 2(\mathrm{FPKM}))-\operatorname{Min}(\log 2$ $($ FPKM $))>2$. The filtered gene set was clustered hierarchically using the gplots package installed under R Bioconductor V2.8 [66], and 22 initial clusters were manually identified. Genes from these clusters were scored as colony, swimming, thecate, colony and swimming, thecate and swimming, and colony and thecate based on their expression patterns (Figure S2 in Additional file 1).

\section{OrthoMCL}

Predicted protein sets for 34 genomes (Table S4 in Additional file 1) were generated from the longest protein greater than 30 amino acids for each gene. Then all-vs-all blastp [41] (E-value < 1E-5) was run on the filtered proteins and the OrthoMCL2 [61] pipeline was used to build ortholog clusters with default parameters.

\section{Reconstructing gene gain and loss in opisthokonts}

To characterize how gene content changed during the evolution of the opisthokonts, ortholog clusters from OrthoMCL2, including single gene clusters, were mapped to a reference phylogeny $[61,62]$ using a Dollo parsimony model [63]. The minimal gene content at each node and the change along the subsequently diverging lineages were then catalogued. The presence or absence of gene families and protein domains mentioned in the text were manually verified using homologs from NCBI homologene and BLASTP and tBLASTn (cutoff e10 ${ }^{-3}$ ) [41].

\section{Ortholog cluster origin enrichment analysis}

Ortholog clusters were annotated as ancient, choanimal, choanoflagellate or S. rosetta-unique based on the cluster member most distantly related to $S$. rosetta. The relative frequencies of phylogenic annotations were calculated for the entire S. rosetta genome (Figure 4a). Expression clusters were tested for phylogenic enrichment by comparing their annotation counts to frequencies for the entire genome. Annotation counts were assumed to follow a multinomial distribution, which was validated through a Monte Carlo simulation (data not shown).

A jackknifing analysis was run to test the sensitivity of phylogenic enrichment to the species included (Figure S10 in Additional file 1); 10,000 trials were run, each with a random set of species. S. rosetta and M. brevicollis were included in all trials. Each of the 32 remaining species had an $80 \%$ probability of being included in any given trial. The OrthoMCL2 algorithm was rerun for each species set to generate new clusters. Annotation frequencies were recalculated for the entire genome and the expression clusters were tested for phylogenic enrichment.

The MCL algorithm was run an additional 19 times to test the sensitivity of the results to the inflation parameter of the MCL algorithm (Figure S11 in Additional file 1). Values for inflation ranged from 1.1 to 3 . All 34 analyzed species were included.

\section{Data availability}

Raw 454 genome sequence data have been submitted to NCBI's Short Read Archive and can be retrieved using the following accession numbers: fragment reads (SRX015529, SRX015528, SRX015527, SRX015526, SRX015525, SRX015524, SRX015523, SRX015522, SRX015521, SRX015515, SRX015514, SRX015512, SRX015511, SRX015503, SRX015502, SRX015499, SRX015498, SRX015486, SRX015485, SRX015484, SRX015483, SRX015482, SRX015457, SRX015456); and 2 to $3 \mathrm{~kb}$ jumping reads (SRX015508, SRX015505, SRX015501, SRX015464, SRX015463, SRX015458). Raw Sanger sequence data have been submitted to NCBI's Trace Archive and can be retrieved using the following search parameters: CENTER_NAME = "BI" and CENTER_PROJECT = "G1237". The genome assembly was submitted to NCBI with accession number ACSY00000000. Genome sequence and transcriptome sequence have been deposited in GenBank under project codes PRJNA37927 and SRP005692, respectively. A genome browser is available at the Broad Institute website [67].

Raw sequence data from Illumina sequencing of celltype enriched transcriptomes has been submitted to NCBI's Short Read Archive using the following accession numbers: RCAM, SRX042054 (NK96-sup - culture enriched for colonial cells grown in the presence of mixed bacterial prey and A. machipongonensis); SwM, SRX042053 (Col-sup - solitary swimming cells grown in the presence of mixed bacterial prey); ThA2, SRX042052 (Pxl-att - culture enriched for solitary attached cells grown only in the presence of A. machipongonensis); ThAM, SRX042051 (NK96-att - culture enriched for solitary attached cells grown in the presence of mixed bacterial prey and A. machipongonensis); ThM, SRX042050 (Colatt - culture enriched for solitary attached cells grown in 
the presence of mixed bacterial prey); RCA1, SRX042049 (colonies - culture enriched for colonial cells grown only in the presence of A. machipongonensis); CCM, SRX042047 (Chains - culture enriched for chain cells grown with mixed bacterial prey); RCA2, SRX042046 (Pxl-sup - culture enriched for colonial cells grown only in the presence of $A$. machipongonensis).

\section{Additional material}

Additional file 1: Figures S1 to S14 and Tables S1 to S8. Figure S1: transcriptional profiling experimental design. Figure $\mathbf{S 2}$ : differentially expressed genes identified by hierarchical clustering. Figure S3: identification of upregulated genes. Figure S4: gene expression correlates with cell type. Figure S5: S. rosetta cadherin expression. Figure S6: Hedgehog signal domain-encoding genes are upregulated in thecate and colonial cell types. Figure S7: protein domain architecture of $S$. rosetta septins. Figure S8: septin sequence conservation. Figure S9: septin gene family phylogeny. Figure S10: ortholog cluster origin enrichment is robust to species composition. Figure S11: ortholog cluster origin enrichment is robust to changes in MCL (Markov Cluster algorithm) species inflation value. Figure S12: expression levels of receptor tyrosine kinase families. Figure S13: the phylogenetic distribution of important metazoan development genes or domains. Figure S14: synteny between the S. rosetta and M. brevicollis genomes. Table S1: S. rosetta and M. brevicollis genome statistics. Table S2: mapping of de novo transcript assembly. Table S3: telomeres predicted in the S. rosetta genome. Table S4: genomes used for comparative genomics. Table S5: Gene Ontology enrichment of novel genes. Table S6: S. rosetta tyrosine kinases. Table S7: phylogenetic distribution of genes upregulated in different cell types. Table S8: genes missing from choanoflagellate.

Additional file 2: Number of ortholog pairs shared between S. rosetta and $M$. brevicollis scaffolds.

Additional file 3: The number of genes present in each of the OrthoMCL ortholog clusters.

Additional file 4: The genes present in each of the OrthoMCL ortholog clusters

Additional file 5: OrthoMCL ortholog clusters predicted to be present in the ancestors reconstructed in Figure 2.

Additional file 6: Kinases identified in the S. rosetta genome.

Additional file 7: Read counts and FPKM values (fragments per kilobase per million reads) for genes encoded by the $S$. rosetta genome

Additional file 8: Differentially expressed genes identified by hierarchical clustering.

Additional file 9: Differentially expressed genes identified by pairwise comparison.

Additional file 10: Differentially expressed genes identified by group comparison.

Additional file 11: Genes identified as upregulated in colonial cells Additional file 12: Genes identified as upregulated in thecate cells. Additional file 13: Genes identified as upregulated in swimming cells. Additional file 14: Blast2GO annotation of S. rosetta genome

Additional file 15: Interpro annotation of $S$. rosetta genome.

\section{Abbreviations}

bp: base pair; Hh: Hedgehog; RTK: receptor tyrosine kinase; TGF: transforming growth factor; TK: tyrosine kinase.

\section{Competing interests}

The authors declare no competing financial interests.

\section{Authors' contributions}

SF and NK conceived the project, SF setup experiments, harvested samples, and prepared DNA and RNA for sequencing. CR managed sequencing and data deposition. SY assembled the genome. HR annotated telomeres. ZC and QD annotated the genome. ZH, DR and SF analyzed annotation. EK and GM annotated and analyzed TKs. EB, JW, and SF analyzed Septins. BH assembled RNA-seq transcripts. ZH and SF analyzed expression data. EK and SF analyzed ortholog and expression patterns. SF and NK wrote the manuscript with significant input from ZC, EK, GM, BH, and CN. All authors read and approved the final manuscript.

\section{Acknowledgements}

We thank the Broad Institute Genomics Platform for sequence data generation. This work was supported by funding from NIH NHGR U54HG003067, NIGMS R01 GM089977 (NK), an American Cancer Society Research Scholar Grant (NK), the Gordon and Betty Moore Foundation Marine Microbiology Initiative (NK), NIH Training Grant T32 HG 00047 (SRF), a National Defense Science and Engineering Graduate Fellowship (DJR), and NHGRI R01 HG004164 (GM). NK is a Fellow in the Integrated Microbial Biodiversity Program of the Canadian Institute for Advanced Research.

\section{Endnotes}

'The term 'Choanozoa,' first coined by Shalchian-Tabrizi et al. [75], refers to the paraphyletic group that contains Capsaspora owczarzaki and choanoflagellates, but excludes metazoans.

\section{${ }^{\mathrm{b}} \mathrm{S}$. rosetta tyrosine kinases}

The S. rosetta genome encodes 469 protein kinases. The 376 serine/ threonine kinases are generally well conserved between $M$. brevicollis and $S$. rosetta (Table S6 in Additional file 1). In contrast, the TKs seem to be more rapidly evolving and show divergent sequences and large numbers of gains and losses (Table S6 in Additional file 1). Comparison of the 93 S. rosetta and $135 \mathrm{M}$. brevicollis TKs suggests a core choanoflagellate tyrosine kinome of approximately 51 kinases, with extensive gains and some losses in the two species. The Fer and FAK kinases appear to have been lost in M. brevicollis, as they are found in S. rosetta, metazoans, and Capsaspora owczarzaki. Fer and FAK both regulate cell adhesion in metazoans, suggesting that $M$. brevicollis has lost some ancestral cell-adhesion functions.

The $S$. rosetta sequences allow an improved classification of both choanoflagellate tyrosine kinomes, resulting in six new families of RTKs (RTKN-T) and the creation of other families (UTKA-H) from many of the previously unique TKs. The additional sequences also indicate orthology between some choanoflagellate and metazoan RTKs: Eph RTKs are found in both species, and several other RTKs are weakly Eph-like. More tentatively, the new RTKS family may be orthologous to the insulin/IGF1R family, although it has only partial similarity to the extracellular regions of metazoan insulin receptors.

The cytoplasmic (non-receptor) TKs are evolutionarily stable between $M$. brevicollis and S. rosetta, with $90 \%$ of kinases in common, the only differences being one extra CTKA and FYTK in M. brevicollis, and the loss of FAK and Fer from M. brevicollis. The large family of 15 HMTKs is completely conserved between the two sequenced choanoflagellate species. The receptor TKs are more evolutionarily dynamic, with eight families specific to M. brevicollis, three families specific to S. rosetta, and only $21 \%$ of all RTKs orthologous between the two sequenced choanoflagellates. Another eight TK families (UTKA-H) could not be classified as cytoplasmic or receptor, and the 'TK-Unique' kinases have no clear homologs between the two species.

As with other genes, we see that $S$. rosetta TKs that are conserved with $M$. brevicollis tend to be more highly expressed in attached cells, while those unique to $\mathrm{S}$. rosetta are more highly expressed in rosette colonies (Figure S12 in Additional file 1).

\section{${ }^{\mathrm{S}} \mathrm{S}$. rosetta cadherin and hedgling protein diversity}

The S. rosetta genome is predicted to encode 29 proteins containing cadherin domains [23], a number that is comparable to the complement of cadherins found in the genomes of $\mathrm{M}$. brevicollis and many animals (including 17 in D. melanogaster and 32 in C. intestinalis) [76]. While cadherins are well known for their roles in animal cell adhesion and intercellular signaling [77], their functions in choanoflagellates are unknown. Two S. rosetta cadherins, PTSG_06458 and PTSG_06068, are upregulated in colonies relative to single cells and an additional six are upregulated in thecate cells relative to colonies (Figure S5 in Additional file 1). 
Two of the six cadherins that are upregulated in thecate cells are homologs of Hedgling. Hedgling proteins are conserved in choanoflagellates, sponges, and cnidaria, and proposed to represent evolutionary antecedents of the metazoan developmental signaling protein $\mathrm{Hh}[2,27]$. Unlike canonical metazoan Hh proteins (which contain an amino-terminal Hh signal domain and a carboxy-terminal autocatalytic HINT domain), Hedgling proteins are large transmembrane proteins that contain an amino-terminal Hh domain, an adjacent WWA domain, and multiple cadherin repeats; some also contain tumor necrosis factor receptor, Furin, and epidermal growth factor domains, although these are not universally conserved $[2,78]$. Prior to the sequencing of the S. rosetta genome, the choanoflagellate Hedglings were the only known non-metazoan Hh-domain containing proteins. With the sequencing of the $S$. rosetta genome, we have discovered five additional non-Hedgling proteins that contain Hh domains. A unifying characteristic of these proteins and all Hedglings, including those from the sponge Amphimedon queenslandica and the cnidarian Nematostella vectensis, is the positioning of the Hh domain immediately adjacent to a WWA domain; this pairing may represent an ancient cassette that was also encoded by the genes from which metazoan $\mathrm{Hh}$ evolved. Four of the Hh signaling domain-containing proteins also have a predicted transmembrane domain and these proteins are all upregulated in thecate cells (Figure S6 in Additional file 1). The remaining three $\mathrm{Hh}$ domain-containing proteins are relatively small, contain an amino-terminal signal sequence, and lack a transmembrane domain; these proteins are consistently upregulated in colonial cells where they may act as secreted ligands. Like $M$. brevicollis, the $S$. rosetta genome also encodes homologs of Patched, the Hh receptor in metazoans, allowing the possibility that the Hh-Patched interaction preceded the origin of metazoans.

\section{Author details}

'Department of Molecular and Cell Biology, University of California Berkeley, Berkeley, CA 94720, USA. 'Broad Institute of MIT and Harvard, Cambridge, MA 02141, USA. ${ }^{3}$ Department of Computational Biology, Genentech, 1 DNA Way, South San Francisco, CA 94080, USA. ${ }^{4}$ Department of Entomology, University of Illinois at Urbana-Champaign, Urbana, IL 61801, USA. ${ }^{5}$ Departement de Biochimie, Universite de Montreal, Montreal, Quebec, Canada.

Received: 13 November 2012 Revised: 30 January 2013 Accepted: 18 February 2013 Published: 18 February 2013

\section{References}

1. Carr M, Leadbeater BS, Hassan R, Nelson M, Baldauf SL: Molecular phylogeny of choanoflagellates, the sister group to Metazoa. Proc Natl Acad Sci USA 2008, 105:16641-16646.

2. King N, Westbrook MJ, Young SL, Kuo A, Abedin M, Chapman J, Fairclough S, Hellsten U, Isogai Y, Letunic I, Marr M, Pincus D, Putnam N, Rokas A, Wright K, Zuzow R, Dirks W, Good M, Goodstein D, Lemons D, Li W, Lyons JB, Morris A, Nichols S, Richter DJ, Salamov A, Sequencing JG, Bork P, Lim WA, Manning G, et al: The genome of the choanoflagellate Monosiga brevicollis and the origin of metazoans. Nature 2008, 451:783-788.

3. Ruiz-Trillo I, Burger G, Holland P, King N, Lang F, Roger A, Gray M: The origins of multicellularity: a multi-taxon genome initiative. Trends in Genetics 2007, 23:113-118.

4. Haeckel E: The gastraea-theory, the phylogenetic classification of the animal kingdom and the homology of the germ-lamelle. Quarterly Journal of Microscopical Science 1874, 14:142-165.

5. Nielsen C: Six major steps in animal evolution: are we derived sponge larvae?. Evol Dev 2008, 10:241-257.

6. Dayel MJ, Alegado RA, Fairclough SR, Levin TC, Nichols SA, Mcdonald K, King N: Cell differentiation and morphogenesis in the colony-forming choanoflagellate Salpingoeca rosetta. Developmental Biology 2011, 357:73-82.

7. Valentine J: Architectures of Biological Complexity. Integr Comp Biol 2003, 43:99-103.

8. Bauer S, Grossmann S, Vingron M, Robinson PN: Ontologizer 2.0-a multifunctional tool for $\mathrm{GO}$ term enrichment analysis and data exploration. Bioinformatics (Oxford, England) 2008, 24:1650-1651.

9. Ashburner M, Ball CA, Blake JA, Botstein D, Butler H, Cherry JM, Davis AP, Dolinski K, Dwight SS, Eppig JT, Harris MA, Hill DP, Issel-Tarver L, Kasarskis A,
Lewis S, Matese JC, Richardson JE, Ringwald M, Rubin GM, Sherlock G: Gene ontology: tool for the unification of biology. The Gene Ontology Consortium. Nature genetics 2000, 25:25-29.

10. Ding $M$, Goncharov $A$, Jin $Y$, Chisholm AD: C. elegans ankyrin repeat protein $\mathrm{VAB}-19$ is a component of epidermal attachment structures and is essential for epidermal morphogenesis. Development (Cambridge, England) 2003, 130:5791-5801.

11. Manning G, Young SL, Miller WT, Zhai Y: The protist, Monosiga brevicollis, has a tyrosine kinase signaling network more elaborate and diverse than found in any known metazoan. Proc Natl Acad Sci USA 2008, 105:9674-9679.

12. Pincus D, Letunic I, Bork P, Lim W: Evolution of the phospho-tyrosine signaling machinery in premetazoan lineages. Proc Natl Acad Sci USA 2008.

13. Wilkinson DG: Eph receptors and ephrins: regulators of guidance and assembly. International review of cytology 2000, 196:177-244.

14. Hartwell LH, Culotti J, Reid B: Genetic control of the cell-division cycle in yeast. I. Detection of mutants. Proc Natl Acad Sci USA 1970, 66:352-359.

15. Nguyen TQ, Sawa H, Okano H, White JG: The C. elegans septin genes, unc59 and unc-61, are required for normal postembryonic cytokineses and morphogenesis but have no essential function in embryogenesis. Journal of cell science 2000, 113:3825-3837.

16. Estey MP, Di Ciano-Oliveira C, Froese CD, Bejide MT, Trimble WS: Distinct roles of septins in cytokinesis: SEPT9 mediates midbody abscission. The Journal of cell biology 191:741-749.

17. Mostowy S, Cossart P: Septins: the fourth component of the cytoskeleton. Nature reviews 13:183-194.

18. Fairclough SR, Dayel MJ, King N: Multicellular development in a choanoflagellate. Current Biology 2010, 20:R875-R876.

19. Knoll AH, Carroll SB: Early animal evolution: emerging views from comparative biology and geology. Science 1999, 284:2129-2137.

20. King N: The unicellular ancestry of animal development. Dev Cell 2004, 7:313-325.

21. True JR, Carroll SB: Gene co-option in physiological and morphological evolution. Annu Rev Cell Dev Biol 2002, 18:53-80.

22. Erwin DH: The Origin of Metazoan Development - a Paleobiological Perspective. Biological Journal of the Linnean Society 1993, 50:255-274.

23. Nichols SA, Roberts BW, Richter DJ, Fairclough SR, King N: Origin of metazoan cadherin diversity and the antiquity of the classical cadherin/ beta-catenin complex. Proc Natl Acad Sci USA 2012.

24. Suga H, Dacre M, de Mendoza A, Shalchian-Tabrizi K, Manning G, RuizTrillo I: Genomic survey of premetazoans shows deep conservation of cytoplasmic tyrosine kinases and multiple radiations of receptor tyrosine kinases. Science signaling 2012, 5:ra35.

25. Li WQ, Young SL, King N, Miller WT: Signaling properties of a nonmetazoan Src kinase and the evolutionary history of Src negative regulation. Journal of Biological Chemistry 2008, 283:15491-15501.

26. Segawa Y, Suga H, Iwabe N, Oneyama C, Akagi T, Miyata T, Okada M: Functional development of Src tyrosine kinases during evolution from a unicellular ancestor to multicellular animals. Proc Natl Acad Sci USA 2006, 103:12021-12026.

27. Adamska M, Matus DQ, Adamski M, Green K, Rokhsar DS, Martindale MQ, Degnan BM: The evolutionary origin of hedgehog proteins. Curr Biol 2007, 17:R836-837.

28. Ruiz-Trillo I, Burger G, Holland PW, King N, Lang BF, Roger AJ, Gray MW: The origins of multicellularity: a multi-taxon genome initiative. Trends Genet 2007, 23:113-118.

29. Nichols SA, Dirks W, Pearse JS, King N: Early evolution of animal cell signaling and adhesion genes. Proc Natl Acad Sci USA 2006, 103:12451-12456.

30. Srivastava M, Simakov O, Chapman J, Fahey B, Gauthier ME, Mitros T, Richards GS, Conaco C, Dacre M, Hellsten U, Larroux C, Putnam NH, Stanke M, Adamska M, Darling A, Degnan SM, Oakley TH, Plachetzki DC, Zhai Y, Adamski M, Calcino A, Cummins SF, Goodstein DM, Harris C, Jackson DJ, Leys SP, Shu S, Woodcroft BJ, Vervoort M, Kosik KS, et al: The Amphimedon queenslandica genome and the evolution of animal complexity. Nature 2010, 466:720-726.

31. Adamska M, Larroux C, Adamski M, Green K, Lovas E, Koop D, Richards GS, Zwafink C, Degnan BM: Structure and expression of conserved Wnt pathway components in the demosponge Amphimedon queenslandica. Evolution \& Development 2010, 12:494-518. 
32. King N, Carroll SB: A receptor tyrosine kinase from choanoflagellates: molecular insights into early animal evolution. Proc Natl Acad Sci USA 2001, 98:15032-15037.

33. Lennon NJ, Lintner RE, Anderson S, Alvarez P, Barry A, Brockman W, Daza R, Erlich RL, Giannoukos G, Green L, Hollinger A, Hoover CA, Jaffe DB, Juhn F McCarthy D, Perrin D, Ponchner K, Powers TL, Rizzolo K, Robbins D, Ryan E, Russ C, Sparrow T, Stalker J, Steelman S, Weiand M, Zimmer A, Henn MR, Nusbaum C, Nicol R: A scalable, fully automated process for construction of sequence-ready barcoded libraries for 454 . Genome biology 2010, 11:R15.

34. Newbler assembler. [http://454.com/products/analysis-software/index.asp].

35. HybridAssemble. [http://www.broadinstitute.org/crd/wiki/index.php/ HybridAssemble].

36. Jaffe DB, Butler J, Gnerre S, Mauceli E, Lindblad-Toh K, Mesirov JP, Zody MC Lander ES: Whole-genome sequence assembly for mammalian genomes: Arachne 2. Genome research 2003, 13:91-96.

37. Haas BJ, Delcher AL, Mount SM, Wortman JR, Smith RK Jr, Hannick LI, Maiti R, Ronning CM, Rusch DB, Town CD, Salzberg SL, White O: Improving the Arabidopsis genome annotation using maximal transcript alignment assemblies. Nucleic acids research 2003, 31:5654-5666.

38. Haas BJ, Salzberg SL, Zhu W, Pertea M, Allen JE, Orvis J, White O, Buell CR, Wortman JR: Automated eukaryotic gene structure annotation using EVidenceModeler and the Program to Assemble Spliced Alignments. Genome biology 2008, 9:R7.

39. Rhind N, Chen Z, Yassour M, Thompson DA, Haas BJ, Habib N, Wapinski I, Roy S, Lin MF, Heiman DI, Young SK, Furuya K, Guo Y, Pidoux A, Chen HM, Robbertse B, Goldberg JM, Aoki K, Bayne EH, Berlin AM, Desjardins CA, Dobbs E, Dukaj L, Fan L, FitzGerald MG, French C, Gujja S, Hansen K, Keifenheim D, Levin JZ, et al: Comparative functional genomics of the fission yeasts. Science 2011, 332:930-936.

40. Haas BJ, Zeng Q, Pearson MD, Cuomo CA, Wortman JR: Approaches to Fungal Genome Annotation. Mycology 2011, 2:118-141.

41. Altschul SF, Gish W, Miller W, Myers EW, Lipman DJ: Basic local alignment search tool. Journal of molecular biology 1990, 215:403-410.

42. Kent WJ: BLAT-the BLAST-like alignment tool. Genome research 2002 12:656-664.

43. Manning G, Whyte DB, Martinez R, Hunter T, Sudarsanam S: The protein kinase complement of the human genome. Science 2002, 298:1912-1934

44. Caenepeel S, Charydczak G, Sudarsanam S, Hunter T, Manning G: The mouse kinome: discovery and comparative genomics of all mouse protein kinases. Proc Natl Acad Sci USA 2004, 101:11707-11712.

45. Bradham CA, Foltz KR, Beane WS, Arnone MI, Rizzo F, Coffman JA, Mushegian A, Goel M, Morales J, Geneviere AM, Lapraz F, Robertson AJ, Kelkar H, Loza-Coll M, Townley IK, Raisch M, Roux MM, Lepage T, Gache C, McClay DR, Manning G: The sea urchin kinome: a first look. Dev Biol 2006, 300:180-193.

46. Manning G, Plowman G, Hunter T, Sudarsanam S: Evolution of protein kinase signaling from yeast to man. Trends Biochem Sci 2002, 27:514.

47. Plowman GD, Sudarsanam S, Bingham J, Whyte D, Hunter T: The protein kinases of Caenorhabditis elegans: a model for signal transduction in multicellular organisms. Proc Natl Acad Sci USA 1999, 96:13603-13610.

48. Manning G, Young SL, Miller WT, Zhai YF: The protist, Monosiga brevicollis, has a tyrosine kinase signaling network more elaborate and diverse than found in any known metazoan. Proc Natl Acad Sci USA 2008, 105.9674-9679.

49. Hunter T, Plowman GD: The protein kinases of budding yeast: six score and more. Trends Biochem Sci 1997, 22:18-22.

50. Banks JA, Nishiyama T, Hasebe M, Bowman JL, Gribskov M, dePamphilis C, Albert VA, Aono N, Aoyama T, Ambrose BA, Ashton NW, Axtell MJ, Barker E, Barker MS, Bennetzen JL, Bonawitz ND, Chapple C, Cheng C, Correa LG, Dacre M, DeBarry J, Dreyer I, Elias M, Engstrom EM, Estelle M, Feng L, Finet C, Floyd SK, Frommer WB, Fujita T, et al: The Selaginella genome identifies genetic changes associated with the evolution of vascular plants. Science 2011, 332:960-963.

51. Bateman A, Birney E, Cerruti L, Durbin R, Etwiller L, Eddy SR, GriffithsJones S, Howe KL, Marshall M, Sonnhammer EL: The Pfam protein families database. Nucleic acids research 2002, 30:276-280.

52. Lupas A, Van Dyke $M$, Stock J: Predicting coiled coils from protein sequences. Science 1991, 252:1162-1164.

53. Loopp: Part of this work was carried out by using the resources of the Computational Biology Service Unit from Cornell University which is partially funded by Microsoft Corporation. 2011.
54. LOOPP.. [http://www.loopp.org]

55. Schrodinger LLC: The PyMOL Molecular Graphics System, Version 1.3r1. Book The PyMOL Molecular Graphics System, Version $1.3 \mathrm{r} 1$ (Editor ed.^eds.). City 2010.

56. Momany M, Pan F, Malmberg RL: Evolution and Conserved Domains of the Septins. The Septins John Wiley \& Sons, Ltd; 2008, 35-45.

57. Sievers F, Wilm A, Dineen D, Gibson TJ, Karplus K, Li W, Lopez R, McWilliam H, Remmert M, Soding J, Thompson JD, Higgins DG: Fast, scalable generation of high-quality protein multiple sequence alignments using Clustal Omega. Molecular systems biology 2011, 7:539.

58. Castresana J: Selection of conserved blocks from multiple alignments for their use in phylogenetic analysis. Molecular biology and evolution 2000, 17:540-552.

59. Guindon S, Lethiec F, Duroux P, Gascuel O: PHYML Online-a web server for fast maximum likelihood-based phylogenetic inference. Nucleic acids research 2005, 33:W557-559.

60. Whelan S, Goldman N: A general empirical model of protein evolution derived from multiple protein families using a maximum-likelihood approach. Molecular biology and evolution 2001, 18:691-699.

61. Li L, Stoeckert CJ Jr, Roos DS: OrthoMCL: identification of ortholog groups for eukaryotic genomes. Genome research 2003, 13:2178-2189.

62. Chen F, Mackey AJ, Stoeckert CJ Jr, Roos DS: OrthoMCL-DB: querying a comprehensive multi-species collection of ortholog groups. Nucleic acids research 2006, 34:D363-368.

63. Le Quesne WJ: The Uniquely Evolved Character Concept and its Cladistic Application. Systematic Biology 1974, 23:513-517.

64. Alegado RA, Brown LW, Cao S, Dermenjian RK, Zuzow R, Fairclough SR, Clardy J, King N: A bacterial sulfonolipid triggers multicellular development in the closest living relatives of animals. eLife 2012, 1: e00013.

65. Djupedal I, Kos-Braun IC, Mosher RA, Soderholm N, Simmer F, Hardcastle TJ, Fender A, Heidrich N, Kagansky A, Bayne E, Wagner EG, Baulcombe DC, Allshire RC, Ekwall K: Analysis of small RNA in fission yeast; centromeric siRNAs are potentially generated through a structured RNA. The EMBO journal 2009, 28:3832-3844.

66. Robinson MD, McCarthy DJ, Smyth GK: edgeR: a Bioconductor package for differential expression analysis of digital gene expression data. Bioinformatics (Oxford, England) 2010, 26:139-140.

67. Origins of Multicellularity Database. [http://www.broadinstitute.org/ annotation/genome/multicellularity project/MultiHome.html].

68. Baldauf SL: The deep roots of eukaryotes. Science 2003, 300:1703-1706.

69. Philippe H, Derelle R, Lopez P, Pick K, Borchiellini C, Boury-Esnault N, Vacelet J, Renard E, Houliston E, Quéinnec E: Phylogenomics Revives Traditional Views on Deep Animal Relationships. Current Biology 2009, 19:706-712.

70. Ruiz-Trillo I, Roger AJ, Burger G, Gray MW, Lang BF: A phylogenomic investigation into the origin of metazoa. Molecular biology and evolution 2008, 25:664-672.

71. Philippe $H$, Brinkmann H, Lavrov DV, Littlewood DTJ, Manuel M, Wörheide G, Baurain D: Resolving difficult phylogenetic questions: why more sequences are not enough. Plos Biol 2011, 9:e1000602.

72. Schierwater B, Eitel M, Jakob W, Osigus H-J, Hadrys H, Dellaporta SL, Kolokotronis S-O, Desalle R: Concatenated analysis sheds light on early metazoan evolution and fuels a modern "urmetazoon" hypothesis. Plos Biol 2009, 7:e20.

73. Dunn C, Hejnol A, Matus D, Pang K, Browne W, Smith S, Seaver E, Rouse G, Obst M, Edgecombe G, Sørensen M, Haddock S, Schmidt-Rhaesa A, Okusu A, Kristensen R, Wheeler W, Martindale M, Giribet G: Broad phylogenomic sampling improves resolution of the animal tree of life. Nature 2008, 452:745-749.

74. Sirajuddin M, Farkasovsky M, Hauer F, Kuhlmann D, Macara IG, Weyand M Stark $H$, Wittinghofer A: Structural insight into filament formation by mammalian septins. Nature 2007, 449:311-315.

75. Shalchian-Tabrizi K, Minge MA, Espelund M, Orr R, Ruden T, Jakobsen KS, Cavalier-Smith T: Multigene phylogeny of choanozoa and the origin of animals. PloS one 2008, 3:e2098.

76. Abedin M, King N: The premetazoan ancestry of cadherins. Science 2008, 319:946-948.

77. Halbleib JM, Nelson WJ: Cadherins in development: cell adhesion, sorting, and tissue morphogenesis. Genes Dev 2006, 20:3199-3214. 
78. Richards G, Simionato E, Perron M, Adamska M, Vervoort M, Degnan B: Sponge Genes Provide New Insight into the Evolutionary Origin of the Neurogenic Circuit. Current Biology 2008, 18:1156-1161.

doi:10.1186/gb-2013-14-2-r15

Cite this article as: Fairclough et al:: Premetazoan genome evolution and the regulation of cell differentiation in the choanoflagellate Salpingoeca rosetta. Genome Biology 2013 14:R15.

Submit your next manuscript to BioMed Central and take full advantage of:

- Convenient online submission

- Thorough peer review

- No space constraints or color figure charges

- Immediate publication on acceptance

- Inclusion in PubMed, CAS, Scopus and Google Scholar

- Research which is freely available for redistribution

Submit your manuscript at www.biomedcentral.com/submit
Ciomed Central 